\title{
146. Studies on the Voltinism in the Silkworm, Bombyx mori L., with Special Reference to the Organs Concerning Determination of Voltinism (A Preliminary Note). ${ }^{1)}$
}

By Kinsaku Hasegawa.

Faculty of Agriculture, Tottcri University. (Comm. by Yô K. OKAdA, M.J.A., Dec. 12, 1951.)

At the meetings of the Sericultural Society of Japan in Tokyo on A.pril 10th, 1950, and in Kyoto on May 6th, 1951, the author reported that the anterior part of the silkworm pupae, particularly the head, was closely related to the determination of voltinism of the eggs to be laid by the resultant moths and that the decapitation of the pupae induced an alteration of voltinism in either case where the silkworms had been determined to lay hibernating or non-hibernating eggs. He (1950) revealed by implanting various organs in or about the head into the isolated abdomens of silkworm pupae that the suboesophageal ganglion played an important rôle in realizing the hibernating property of the eggs to be laid by the moth abdomens formed in the isolated pupal abdomens.

In 1951, further experiments were performed in order to ascertain the function of the suboesophageal ganglion in the mechanism involved in the determination of voltinism and to find out whether the function of the ganglion is species-specific or not. The present paper embodies the results of the experiments. A complete account of the results so far secured will appear in a paper which will be published in the Journal of the Faculty of Agriculture of the Tottori University.

Experiments: As material were used the silkworms which were determined to produce hibernating eggs (Jap.No.12, Jap.No. 115, Jap. No.115 $\times$ Jap.No.12, Sy and Orange) as well as those destined to produce non-hibernating eggs (Ringetu, N4 and Cambodge). Besides them, under-mentioned wild silkworms were also employed. Various organs from the domesticated silkworms and Antheraea yamamai and Dictyoploca japonica which are all the producers of hibernating eggs, and of $A$. pernyi which passes winter in nature in pupal stage were transplanted into the fourth abdominal segment of the silkworm larvae determined to lay non-hibernating eggs. The corresponding organs of the recipients were left untouched.

A representative example of the results of the experiments is presented in Table 1.

1) The present work was partly supported by a grant in aid of Fundamental Scientific Research of the Educational Department. 


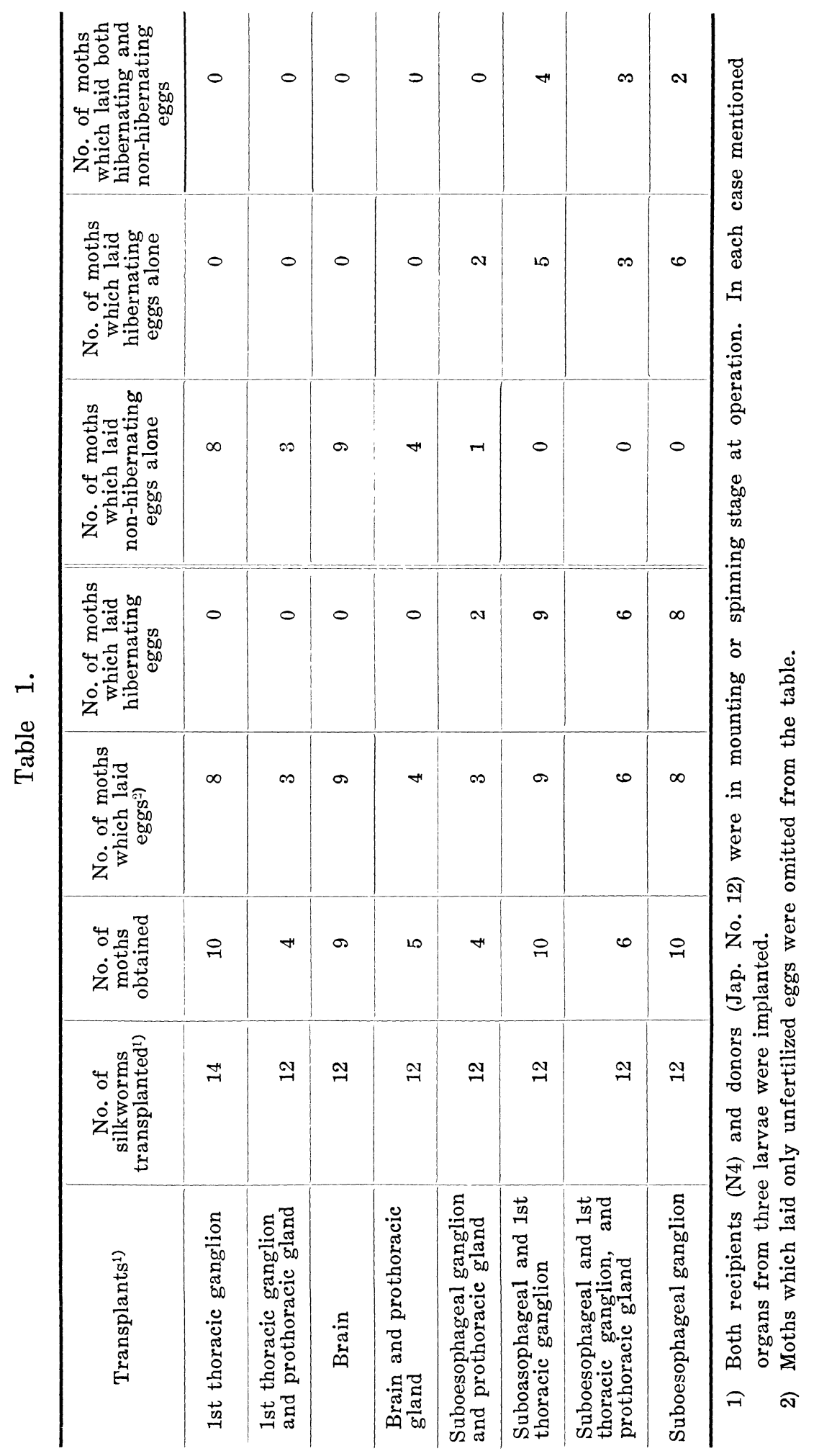


From the table it is seen that if the silkworm larvae which had been determined to lay non-hibernating eggs received as implants the suboesophageal ganglia of the hibernating-egg producer larvae, the voltinism of the eggs to be produced by the resulting moths was altered, i.e., all the moths except one laid hibernating eggs instead of non-hibernating ones. Transplantation of the first thoracic ganglion, brain or prothoracic gland never yielded similar results. These results agree well with those of the author's previous experiments with isolated abdomens carried out in 1950.

Transplants of the suboesophageal ganglia from the larvae of hibernating-egg producer silkworm on the third day of the third instar or older, and of those from the pupae or from the moths ${ }^{2)}$ were also functional in altering non-hibernating eggs into hibernating ones. The recipients at different stages between the third day of the fourth instar and the third day of the pupal stage were affected. Although in the experiments three or four suboesophageal ganglia were implanted, only one was found to be sufficient to produce the effect.

A question arises here whether or not the suboesophageal ganglion of non-hibernating-egg producer plays a part in the determination of voltinism. The author tried to ascertain this by transplanting the ganglia of non-hibernating-egg producers into nonhibernating-egg producers. The results are shown in Table 2. Some moths emerging from the pupae which had received the suboesophageal ganglion alone or together with the brain laid hibernating eggs. Thus the transplants of the suboesophageal ganglia of nonhibernating-egg producers and of hibernating egg-producers functioned similarly although the effect of the former was weaker than that of the latter.

Next step was to see whether the effect of the suboesophageal ganglion was species-specific or not. For this purpose, the suboesophageal ganglia, brains or the first thoracic ganglia of the wild lepidopterans, Antheraea yamamai (the prepupae and moths), $A$. pernyi (the fifth instar larvae) and Dictyoploca japonica (the moths) were transplanted into the silkworms determined to produce nonhibernating eggs. It was found that the brains and the first thoracic ganglia of these forms did not alter the property of eggs to be produced, but the suboesophageal ganglia altered predetermined non-hibernating property of eggs. The potency of the ganglia was not the same in different species. It is worth saying here that the effect of the suboesophageal ganglion of $A$. pernyi, an

2) In the pupae and moths of the silkworm, the suboesophageal ganglion is closely fused to the brain unlike that of the larvae or prepupae. 


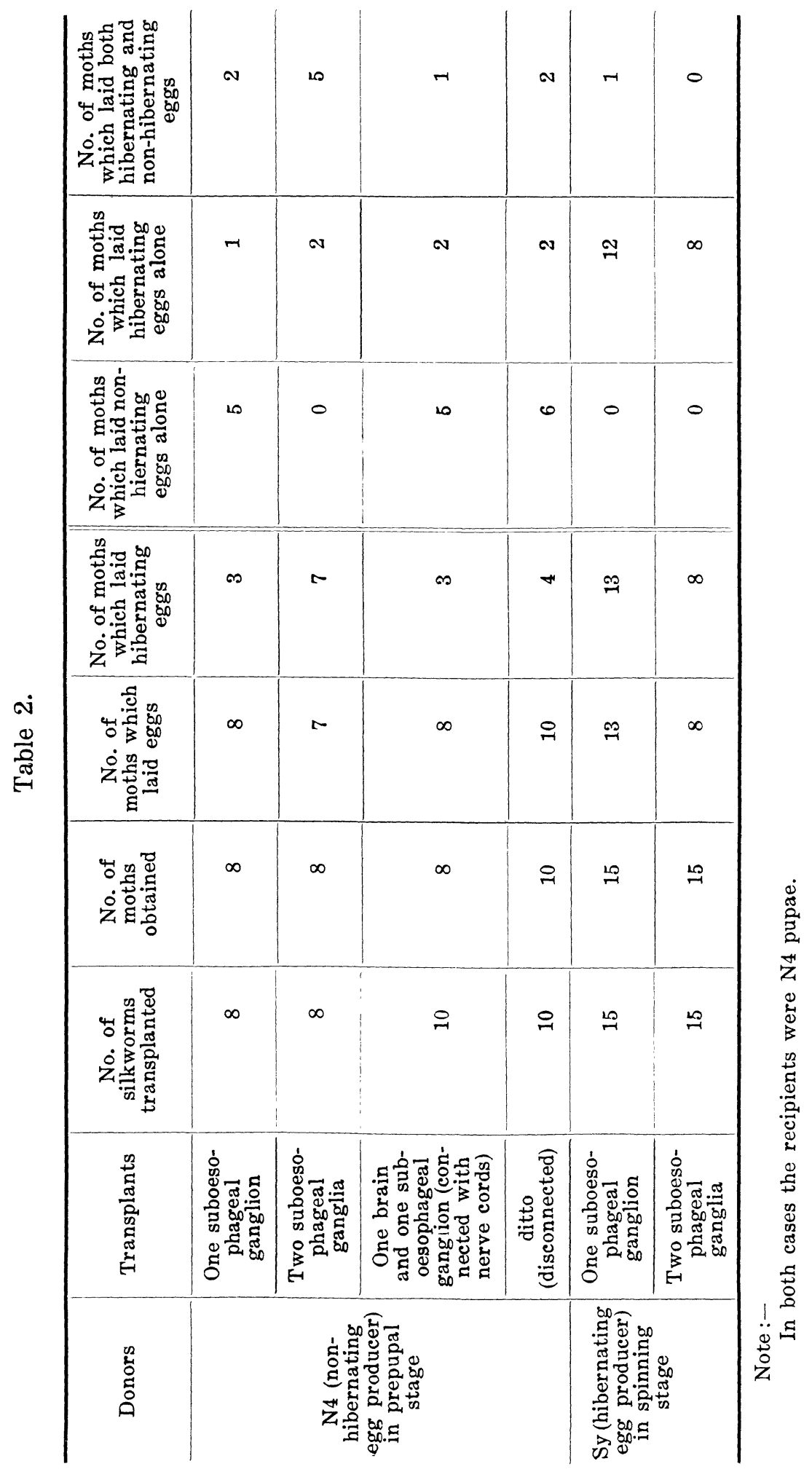


insect which normally passes winter at pupal stage, was as potent as that of domesticated silkworm which was destined to lay hibernating eggs.

Discussion: Judging from the results of the experiments mentioned above, it is highly probable that the suboesophageal ganglion is a centre determining the voltinism of silkworm eggs. It seems likely that in the hibernating-egg producer the suboesophageal ganglion determines the property of ovarian eggs, while in the nonhibernating-egg producer the effect of the ganglion on the eggs may not be strong enough or the effect may be nullified by some unknown factors. Since the function of the suboesophageal ganglion is undoubtedly humoral in nature the ganglion seems to secrete a hormonic substance controlling the hibernating property of the ovarian eggs. A.s stated above, even the ganglion of the third instar, if implanted into the fifth instar larvae, is able to exert its effect on non-hibernating eggs. As to the question of from what stage the substance is normally released the results of the experiments performed in 1950, employing isolated abdomens of the silkworm pupae which were ligated immediately after pupation at the level between the third and fourth abdominal segments, provide an answer. Whereas hibernating eggs (together with the non-hibernating ones) were frequently formed in the isolated abdomens from the pupae of the hibernating-egg producers, almost all of the abdomens from the pupae of the non-hibernating-egg producers brought about nonhibernating eggs. Therefore, the suboesophageal ganglion seems to begin to secrete the active factor from the beginning of the pupal stage.

It is biologically significant to determine whether or not the implanted suboesophageal ganglion influences on the duration of larval life of the silkworm and exerts any influence upon the offspring. The duration of the fifth instar was quite the same in the larvae transplanted on the third day of the fourth instar with the suboesophageal ganglia and in the larvae given the first thoracic ganglia both from hibernating-egg producers, although it was slightly longer than that of the non-operated ones. Accordingly the suboesophageal ganglion seems to exert no noticeable influence upon the duration of larval life. The hibernating eggs laid by the moths bearing the implants of the suboesophageal ganglion, if refrigerated and hydrochlorized as usual, hatched out normally. The voltinism and other characters of the race were not at all disturbed.

Finally, it was demonstrated that the suboesophageal ganglia of the wild lepidopterans function like those of domesticated silkworms in controlling the hibernating property of silkworm eggs regardless of the hibernating habits of the insects in nature. These findings lead us to the conclusion that the hormone of the ganglion is not species-specific in activity. 